\title{
Altered lipid raft-associated signaling and ganglioside expression in T lymphocytes from patients with systemic lupus erythematosus
}

\author{
Elizabeth C. Jury, ${ }^{1}$ Panagiotis S. Kabouridis, ${ }^{2}$ Fabian Flores-Borja, ${ }^{2}$ Rizgar A. Mageed, ${ }^{2}$ \\ and David A. Isenberg 1 \\ ${ }^{1}$ Centre for Rheumatology, Royal Free and University College Medical School, University College London, London, United Kingdom. ${ }^{2 B}$ Bone and Joint \\ Research Unit, Barts and The London, Queen Mary's School of Medicine and Dentistry, Charterhouse Square, London, United Kingdom.
}

\begin{abstract}
Systemic lupus erythematosus (SLE) is characterized by abnormalities in T lymphocyte receptor-mediated signal transduction pathways. Our previous studies have established that lymphocyte-specific protein tyrosine kinase (LCK) is reduced in T lymphocytes from patients with SLE and that this reduction is associated with disease activity and parallels an increase in LCK ubiquitination independent of $T$ cell activation. This study investigated the expression of molecules that regulate LCK homeostasis, such as CD45, C-terminal Src kinase (CSK), and c-Cbl, in lipid raft domains from SLE T cells and investigated the localization of these proteins during $T$ cell receptor (TCR) triggering. Our results indicate that the expression of raft-associated ganglioside, GM1, is increased in T cells from SLE patients and LCK may be differentially regulated due to an alteration in the association of CD45 with lipid raft domains. CD45 tyrosine phosphatase, which regulates LCK activity, was differentially expressed and its localization into lipid rafts was increased in $T$ cells from patients with SLE. Furthermore, T cells allowed to "rest" in vitro showed a reversal of the changes in LCK, CD45, and GM1 expression. The results also revealed that alterations in the level of GM1 expression and lipid raft occupancy cannot be induced by serum factors from patients with SLE but indicated that cell-cell contact, activating aberrant proximal signaling pathways, may be important in influencing abnormalities in $T$ cell signaling and, therefore, function in patients with SLE.
\end{abstract}

\section{Introduction}

Systemic lupus erythematosus (SLE) is a multisystem autoimmune rheumatic disease characterized by the production of high levels of autoantibodies against nuclear antigens resulting at least in part from a dysregulated $\mathrm{T}$ lymphocyte response to autoantigens (1). Current evidence suggests that a central role in initiating disease is played by underlying defects in T lymphocyte antigen receptor-mediated signaling that lead to B cell hyper-responsiveness, increased apoptosis, skewed cytokine production, and breakdown of immunological tolerance (reviewed in refs. 2, 3).

The organization of signaling molecules into discrete membrane-associated microdomains, called lipid rafts, is vital for regulation of $\mathrm{T}$ lymphocyte activation pathways $(4,5,6)$. Raft domains provide an environment favoring immunoreceptor signal transduction, and their disruption, including the abnormal displacement of signaling molecules, has been shown to interrupt intracellular signaling pathways and affect T cell function (7-11).

Nonstandard abbreviations used: British Isles Lupus Assessment Group (BILAG); cholera toxin subunit B (CTB); CSK-binding protein (Cbp); C-terminal Src kinase (CSK); indodicarbocyanine (Cy5); lymphocyte-specific protein tyrosine kinase (LKC); mean fluorescence intensity (MFI); PBS with $0.5 \%$ fish gelatin and $0.3 \%$ saponin (BB-Sap); phycoerythrin (PE); protein tyrosine kinase (PTK); protein tyrosine phosphatase (PTP); rheumatoid arthritis (RA); 0.3\% saponin in SB (SB-Sap); staining buffer (SB); systemic lupus erythematosus (SLE); T cell receptor (TCR); tritiated thymidine $\left(\left[{ }^{3} \mathrm{H}\right] \mathrm{TdR}\right)$.

Conflict of interest: The authors have declared that no conflict of interest exists.

Citation for this article: J. Clin. Invest. 113:1176-1187 (2004).

doi:10.1172/JCI200420345.
This suggests that a change in the properties or composition of lipid rafts may lead to inappropriate $\mathrm{T}$ lymphocyte signaling and ultimately to the development of pathological conditions, including autoimmunity $(12,13)$.

Antigen-mediated $\mathrm{T}$ cell signaling events are initiated by ligation of the T cell receptor (TCR). This leads to a rapid increase in protein tyrosine phosphorylation by recruitment of proximal protein tyrosine kinases (PTKs), the Src family, and syk/Zap-70 kinases, which are responsible for the initiation and amplification of the activation signal (reviewed in ref. 14). The regulation of lymphocyte-specific protein tyrosine kinase (LCK) is critical for maintaining the resting state of $\mathrm{T}$ cells and for initiating the activation of signaling cascades, and in this respect its location to lipid rafts is essential. LCK conformation and kinase activity are regulated by the phosphorylation of two important tyrosine sites: an autophosphorylation site (Tyr394) and an opposing negative regulatory site (Tyr505). Phosphorylation of the negative regulatory site induces the inactive or "closed" LCK conformation (15). There is good evidence that LCK tyrosine phosphorylation is controlled by the reciprocal action of the protein tyrosine phosphatase (PTP) CD45 and the PTK C-terminal Src kinase (CSK) (16). CD45 can positively and negatively regulate the activity of LCK by controlling the phosphorylation of the regulatory tyrosine residues (Tyr394 and Tyr505). In the resting T cell, CD 45 maintains LCK in a primed state ready to respond to TCR activation by dephosphorylating Tyr505. CD45 is preferentially excluded from lipid rafts, however, and regulates LCK activity by its proximity to raft domains (17, 
18). During TCR engagement, CD45 is excluded to the periphery of the immunological synapse and is thus excluded from contact with raft-associated LCK. The action of CD45 on LCK phosphorylation is balanced by CSK, a soluble PTK that interacts with a raftbound adapter protein, CSK-binding protein (Cbp, also known as PAG), thus regulating LCK activity in lipid raft domains (19-22). Furthermore, there is recent evidence that $\mathrm{Cbl}$, an E3 ubiquitin ligase, plays an important regulatory role in the immune response, and it has more recently been associated with negative regulation of active $\operatorname{LCK}(23,24)$. Maintenance of the PTK/PTP balance is thus centered in lipid raft domains, and this allows the immune system to respond rapidly to foreign antigen; an imbalance in this system may lead to inappropriate $T$ cell activation, including the initiation of autoimmune responses (25).

We have shown recently that $\mathrm{T}$ lymphocytes from patients with SLE have reduced expression of LCK that is independent of treatment regime but is associated with disease activity (26). We have also observed an increase in protein ubiquitination, including LCK ubiquitination, in T cells from SLE patients, which was independent of $\mathrm{T}$ cell activation. These results are consistent with a recent report describing reduced expression of $\mathrm{CD} 3-\zeta$ chain associated with increased CD3- $\zeta$ ubiquitination in SLE T cells (27). The purpose of our study here was to investigate whether expression of molecules that regulate LCK homeostasis, such as CD45, CSK, and $\mathrm{c}-\mathrm{Cbl}$, is altered in lipid raft domains from SLE T cells and to investigate the localization of these proteins during TCR triggering. Our results indicate that the proportion of the plasma membrane adopting a lipid raft conformation, measured by binding of cholera toxin subunit B (CTB) to the GM1 component of lipid rafts, is increased in $\mathrm{T}$ cells from patients with SLE. The results also show that LCK may be differentially regulated due to alteration of the association of CD45 with lipid raft domains. Furthermore, our results indicate that TCR activation can trigger alterations in GM1 expression and lipid raft occupancy in SLE T cells, confirming the idea that intracellular signaling pathways are abnormal in these patients.

\section{Results}

$T$ cells from patients with SLE exhibit increased $C T B$ binding and changes in membrane localization of proximal signaling molecules. Our previous studies demonstrated reduced levels of LCK in T cell lipid rafts in SLE. To investigate further the reasons underlying the reduction in LCK, we examined the association between lipid rafts and proximal $\mathrm{T}$ cell signaling molecules by confocal microscopy. To visualize rafts, we stained cells with fluorescence-labeled CTB, which binds the raft-associated glycosphingolipid GM1, previously shown to be a reliable marker for the detection of lipid raft domains (28). A detailed exami-

nation of microscopy images of $\mathrm{T}$ cells from 12 patients with SLE, 8 healthy controls, and 6 patients with rheumatoid arthritis (RA) revealed an increased intensity of CTB binding in T cells from patients with SLE compared with that of T cells from the controls, indicating an increased proportion of the plasma membrane adopts a lipid raft structure. Figure $1 \mathrm{~A}$ shows a representative example of T cells from patients with SLE and healthy controls incubated with CTB-phycoerythrin (CTB-PE) to reveal lipid rafts. The intensity of CTB-PE fluorescence for each image is shown in the accompanying graph displaying mean fluorescence intensity (MFI) measured in arbitrary units (Figure 1A, lower panel). Furthermore, the increase in binding of CTB to GM1 appeared to be associated with reduced LCK/lipid raft colocalization in $\mathrm{T}$ cells from patients with SLE (Figure 1B). When areas of colocalization between LCK and lipid rafts were examined, we observed a reduced number of $\mathrm{T}$ cells exhibiting substantial areas of convergence in SLE patients (Figure 1B, lower panel). This reduction was significant compared with the LCK/lipid raft colocalization seen in $\mathrm{T}$ cells from normal healthy controls $(P=0.01)$ (Figure 1B, lower panel) and patients with RA $(P=0.004)$ (data not shown), thus confirming our previous observations (26). No correlation was seen between increased GM1 expression and disease activity, however, as we observed with LCK expression previously.

Since LCK expression and ubiquitination is altered in SLE T cells (26), we used confocal microscopy to investigate lipid raft association of CSK, c-Cbl, and CD45, proteins that regulate LCK function in $\mathrm{T}$ cells. No significant differences were observed when we examined the localization of CSK and c-Cbl (Figure 2, A, B, and D) in T

A

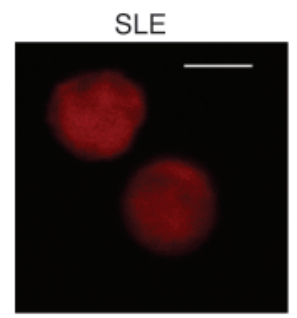

CTB-PE
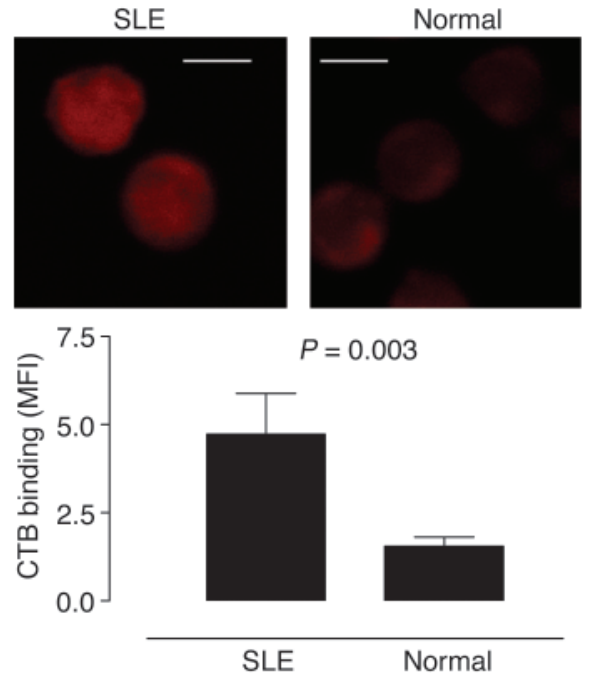

Figure 1

Increased GM1 expression and reduced colocalization of LCK with lipid raft (LR) domains in T cells from patients with SLE. Purified T cells from 12 patients with SLE, 8 healthy controls, and 6 patients with RA were fixed, made permeable, and stained for LCK-FITC by indirect immunofluorescence; lipid rafts were visualized using PE-conjugated CTB. Cells were viewed by confocal microscopy, and images were analyzed for individual LCK or CTB staining patterns and for areas of colocalization (yellow color); quantitative results are based on the "reading" of an average of 50 cells for each sample. (A) Representative experiment showing CTB binding in SLE T cells compared with control T cells (Normal) (upper panels). Semiquantitation of CTB binding is shown using MFI ( \pm SEM); five images were analyzed from five patients with SLE and four healthy controls (lower panel). (B) LCK/CTB overlay images comparing SLE and normal T cells (Normal) (upper panels). Quantitative results showing the percent of $\mathrm{CD}^{+}$cells displaying areas of LCK/LR colocalization in SLE patients and healthy controls; each symbol represents one patient with an average of 50 cells analyzed (lower panel). Scale bars, $5 \mu \mathrm{m}$. 
A СТВ
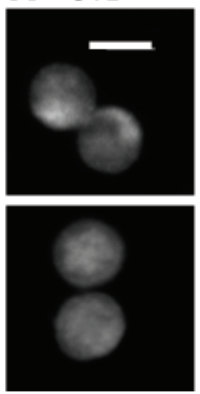

B CTB
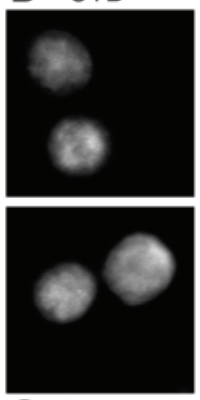

C CTB
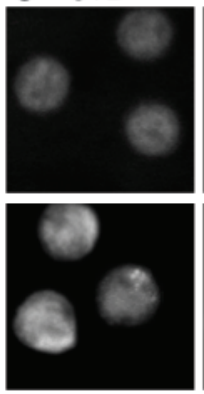

CSK
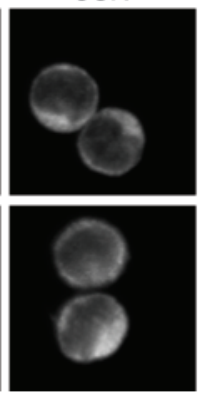

c-Cbl
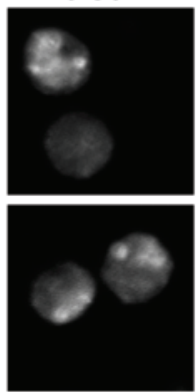

CD45

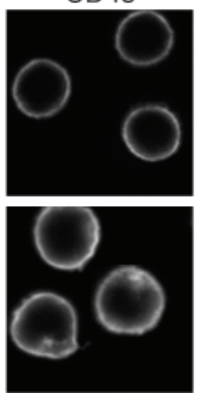

Merge
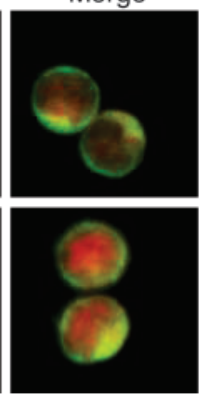

Merge
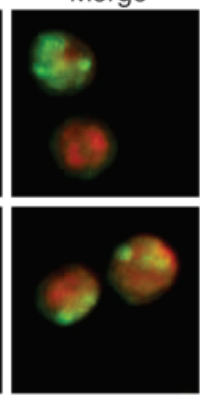

Merge

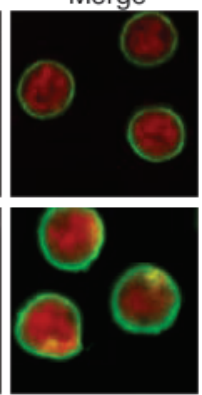

D

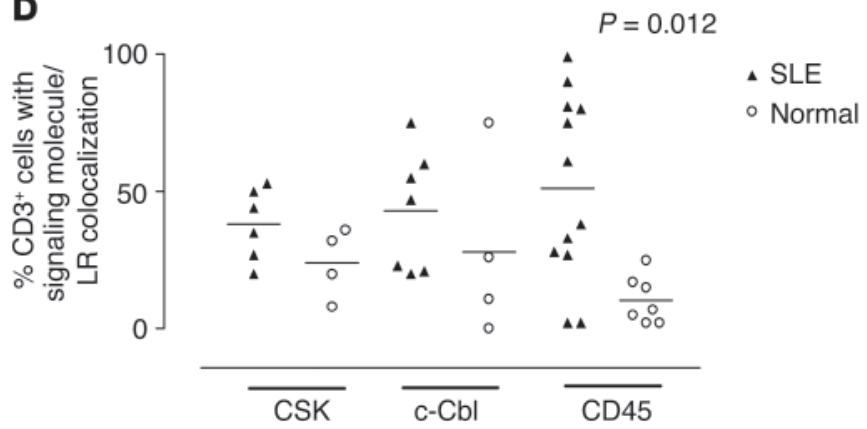

E

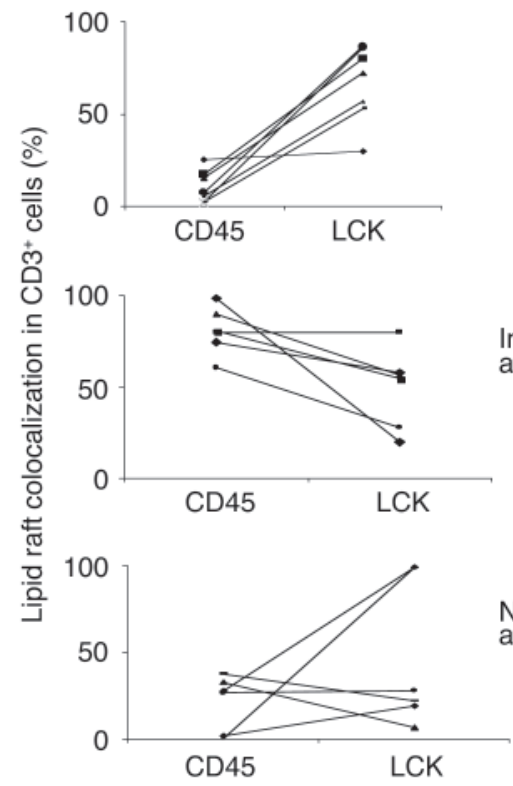

Normal
SLE

Increased CD45/raft association

Figure 2

Altered association of CD45 with LR domains in T cells from patients with SLE. Purified T cells from 12 patients with SLE and 8 healthy controls were examined as described in Figure 1 for CSK, c-Cbl, and CD45. (A-C) Representative experiment showing colocalization of CSK (A), c-Cbl (B), and CD45 (C) to LR domains in SLE and normal T cells (Normal). Scale bars, $5 \mu \mathrm{m}$. (D) Quantitative results showing the percent of CD3+ cells displaying areas of CSK, c-Cbl, and CD45 colocalization with lipid rafts in patients with SLE and healthy controls (Normal). On average, 50 cells were analyzed for each individual sample. (E) Correlation between CD45 and LCK colocalization with rafts domains in individual samples; on average, 50 cells were assessed from each patient or control. T cells from normal controls (top panel), patients with SLE with an increased CD45/LR association (middle panel), and SLE patients with normal CD45/LR colocalization (bottom panel).

cells from patients with SLE compared with those from normal controls. However, CD45 had a significantly increased association with lipid raft domains in approximately $50 \%$ of patients with SLE $(P=0.012)$ (Figure 2, C and D). This observation was not associated with disease activity, treatment regime, or level of expression of the $\mathrm{T}$ cell activation marker CD69 (MFI measured by flow cytometry) (data not shown). There was only a small increase in the number of $\mathrm{CD} 9^{+} \mathrm{T}$ cells in the SLE cohort compared with normal controls (data not shown). The correlation between CD45 and LCK colocalization with rafts domains in individual samples was measured. T cells from 12 patients with SLE and 8 healthy controls were stained with either CD45/CTB or LCK/CTB. Samples were analyzed by confocal microscopy; on average, $50 \mathrm{~T}$ cells from each sample were counted and the number of T cells with LCK/lipid raft or CD45/lipid raft colocalization was assessed. T cells from normal controls had low levels of CD45/lipid raft colocalization and high levels of LCK/lipid raft colocalization (Figure 2E, top panel). In patients with SLE, T cells with an increased CD45/lipid raft association exhibited reduced LCK/lipid raft colocalization (Figure 2E, middle panel), whereas $T$ cells from SLE patients with normal CD45/lipid raft colocalization patterns displayed mixed results. Two patients had increased LCK in raft domains, but four patients displayed reduced levels of LCK in lipid rafts (Figure 2E, bottom panel). These data suggest that reduced LCK levels are accompanied by alterations in CD45 location with regard to lipid raft occupancy. Hence, a significant proportion of patients with SLE have T cells that exhibit increased expression of the lipid raft-associated glycosphingolipid GM1, as determined by CTB binding, together with an increased raft association of the PTP CD45 and reduced raft expression of LCK.

SLE T cells have increased raft-associated CD45 and increased levels of active LCK. Current evidence suggests that CD45 is largely excluded from membrane lipid microdomains $(16,18,28,29)$. The expression of CD45 isoforms on $\mathrm{T}$ cell subpopulations in normal and 

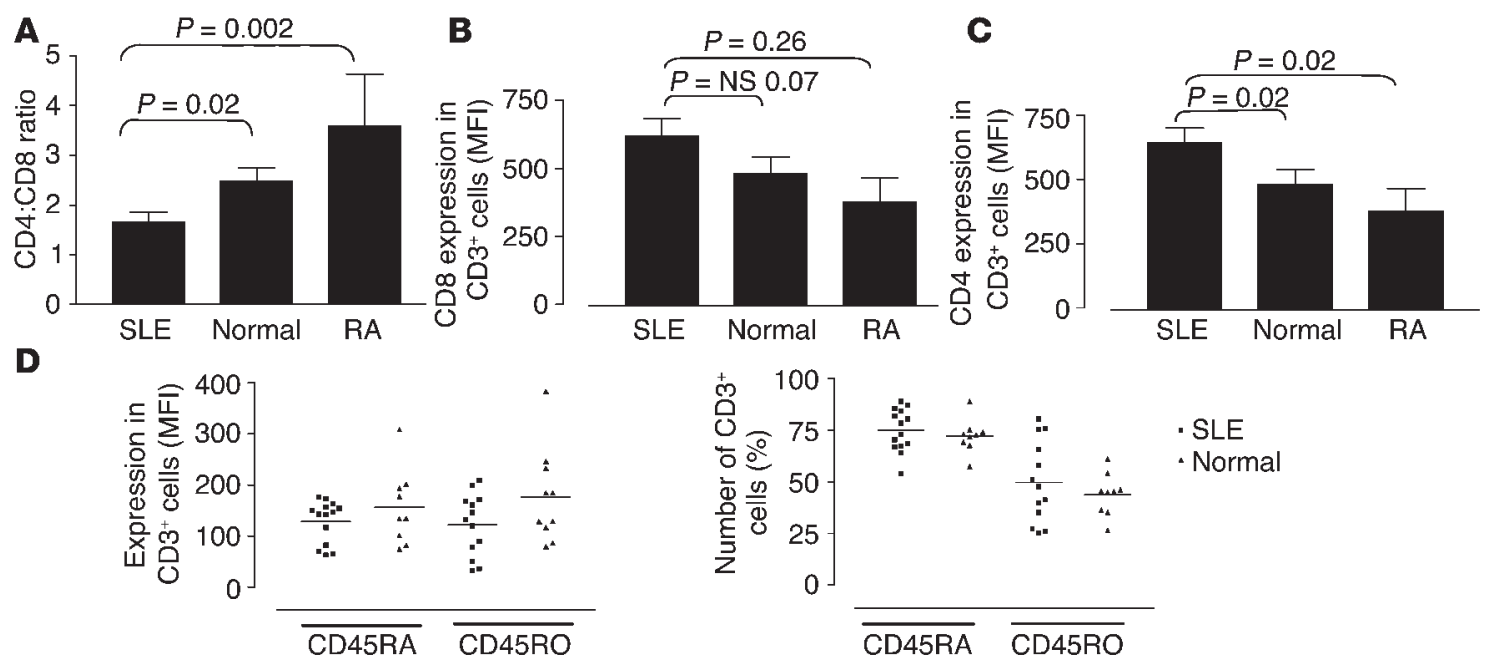

Figure 3

T cells from patients with SLE have an altered phenotype. T lymphocytes from 14 patients with SLE, 12 healthy controls (Normal), and 6 patients with RA were analyzed for expression of CD4, CD8, CD45RA, and CD45RO by flow cytometry. (A) The relative proportions of T cells expressing CD4 or CD8 were expressed as a ratio (CD4:CD8). (B and C) The level of expression of both CD8 (B) and CD4 (C), as determined by MFI, on T cells from patients with SLE or RA and from healthy controls. (D) The level of expression of CD45RA and CD45RO (MFI) on CD3+ cells (left panel) and the number (\%) of $\mathrm{CD}^{+}$cells expressing CD45RA and CD45RO (right panel). All results are expressed as mean \pm SEM.

SLE T cells was assessed by flow cytometry. T lymphocytes from 14 patients with SLE, 12 healthy controls, and 6 patients with RA were analyzed for expression of CD4, CD8, CD45RA, and CD45RO. Patients with SLE had a significantly reduced CD4:CD8 ratio compared with that of healthy controls and patients with RA $(P=0.02$ and $P=0.002$, respectively) (Figure 3A), as described previously (30). The level of expression of both CD4 and CD8 on SLE T cells, as determined by MFI, was increased compared with healthy controls and patients with RA (CD4, $P=0.02$ and $P=0.024$; CD8, $P=0.07$ and $P=0.026$ respectively) (Figure $3, \mathrm{~B}$ and $\mathrm{C}$ ). The contribution of the skewed CD4:CD8 ratio to the pathology of the disease remains unclear. However, when the number of T cells expressing CD45RA and CD45RO were examined, no significant differences were observed between patients with SLE and controls (Figure 3D).

Next we examined the expression of CD45 in T cell raft and nonraft fractions from patients with SLE and compared these results with those of controls. In the non-raft fraction, we observed a significantly reduced expression of CD45 in patients with SLE compared with that of healthy controls $(P=0.02)$ (Figure $4, \mathrm{~A}$ and $\mathrm{B})$. This result shows that in addition to LCK, regulation of CD45 is also altered in $\mathrm{T}$ cells from patients with SLE. The reduction in CD45 expression corresponded to significantly reduced expression of both LCK $(P=0.04)$ and $\mathrm{c}-\mathrm{Cbl}(P=0.02)$, which is shown to mediate ubiquitination of $\operatorname{LCK}(23,24)$. In contrast, expression of CSK was similar in all groups (Figure 4, A and B). The actin Western blot showed that equal protein was analyzed in each sample.

When we examined the expression of CD45 in lipid rafts by Western blotting, we observed an increase in the level of CD45 in SLE T cells compared with that of controls, in agreement with the confocal microscopy results (Figure 4C). CD45 dephosphorylates the inhibitory tyrosine (Tyr505) of LCK, thus allowing LCK to adopt an "open-active" conformation. We examined the activity of LCK in lipid raft fractions by Western blotting using antibodies recognizing the active (phospho-Src pY414) and inactive (phospho-LCK pY505) forms of the molecule. T cells from patients with SLE with an increased expression of CD45 in raft domains showed an increase in the active form of LCK and reduced expression of the inactive form (Figure 4C). The increased interaction between CD45 and LCK in T cells from patients with SLE compared with that of controls was verified by immunoprecipitation of LCK and subsequent analysis of CD45 coprecipitation by Western blot. The results revealed an increased association of CD 45 with LCK in T cells from lupus patients compared with those from controls (Figure 4D). These data support the observation that the distribution and expression of CD45 is altered in T cells from lupus patients and this appears to affect the activity of LCK.

Alterations in T cell lipid raft and LCK expression are reversed after overnight "rest." The observed changes in GM1, LCK, and CD45 expression could reveal an inherent defect of SLE T cells, or alternatively these changes could be imposed on the cells by in vivo factors. To distinguish between these two alternatives, we allowed ex vivo T cells from patients with SLE and controls to "rest" for 24 hours in culture medium. Ex vivo and "rested" cells were analyzed for GM1 expression by CTB binding using confocal microscopy and for LCK expression by flow cytometry. The overexpression of GM1 was reversed in "rested" SLE T cells, whereas in control T cells, no change was observed (Figure 5A). The localization of CD45 and LCK to lipid raft domains was assessed in four patients with SLE and four healthy controls. These experiments revealed reduced CD45/lipid raft association and increased LCK/lipid raft association in the quiescent SLE T cells. No changes were seen in T cells from controls (Figure 5, B and C). These results were verified when LCK levels in T cells from patients with SLE and controls were analyzed by flow cytometry ex vivo and after overnight "rest." Thus, LCK levels recovered in lupus T cells, while no change was seen in T cells from healthy volunteers and patients with RA (Figure 5D). The possibility that these results were being affected by enhanced apoptosis of SLE T cells is not likely to explain the changes seen. Annexin V binding was used to assess the level of $\mathrm{T}$ cell apoptosis in the "rested" cells (Figure 5E). The number ex vivo 
A T cell non-raft fraction

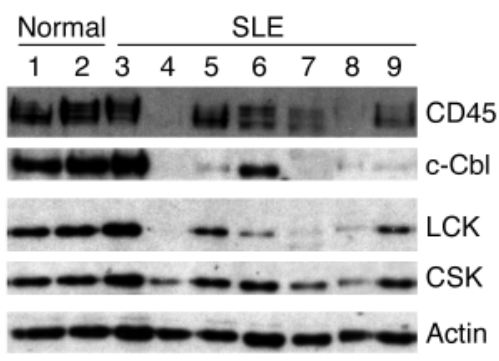

B

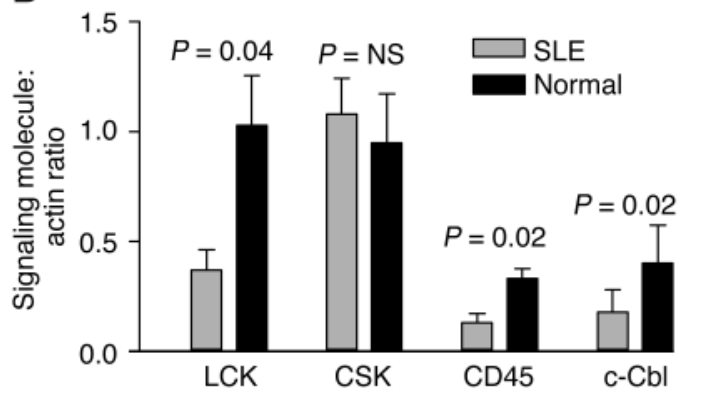

C T cell raft fraction

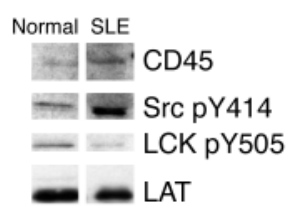

D

Figure 4

CD45 has an altered pattern of expression and membrane localization in T cells from patients with SLE. Lipid raft and non-raft fractions were prepared from T cells from patients with SLE and controls (Normal). Fractions were separated by $8 \%$ SDS-PAGE and analyzed by Western blotting. (A) A representative experiment showing T cell non-raft fractions from patients with SLE and healthy controls analyzed by Western blot for CD45, LCK, c-Cbl, and CSK. Actin was used as a control for equal protein loading. (B) Graph displaying cumulative semiquantitative results for CD45, CSK, c-Cbl, and LCK expression in T cell non-raft fractions from ten SLE patients and five healthy controls. The relative levels of LCK, CSK, CD45, and $\mathrm{c}-\mathrm{Cbl}$ were estimated by comparison of the intensity of each band to that of the actin control. (C) Western blot representative of three experiments showing expression of CD45, active LCK (Src pY414), and inactive LCK (LCK pY505) in T cell lipid rafts. LAT expression is not altered in SLE T cells (26) and is used as a marker of equal protein loading. (D) LCK was immunoprecipitated from $50-\mu \mathrm{g}$ fractions of whole-T cell lysates for patients with SLE and controls (Normal and RA). Immunoprecipitates (IP) were analyzed by Western blot and probed for CD45 and LCK.

$T$ cells expressing annexin $V$ was increased in patients with SLE compared with that of normal controls; after overnight "rest" in complete medium, the levels of annexin V binding remained constant in the SLE T cells but were increased in T cells from normal controls. The reason for the increased level of annexin $\mathrm{V}$ binding in quiescent normal $\mathrm{T}$ cells is not known; however, the results indicate that changes observed in lipid raft occupancy are not due to cells undergoing apoptosis. Thus, these results suggest that the alterations observed in T cells from patients with SLE may be due in part to their exposure to in vivo factors, which could be either soluble serum factors or cell-cell contact.

SLE serum cannot sustain the increased expression of raft-associated GM1 or reduced LCK levels in SLE T cells in vitro. To determine if increased expression of GM1 or reduced LCK expression in SLE T cells is due to soluble serum factors, we assessed T cells from patients with SLE for 72 hours with autologous serum, IgG-depleted autologous serum (to remove autoantibodies), and sera from healthy controls (method modified from ref. 31). Concurrently, T cells from healthy controls were cultured for 72 hours with autologous serum and sera from patients with SLE. SLE T cells showed increased binding of CTB to GM1 ex vivo, as observed previously (shown in Figure $1 \mathrm{~A}$ ), which returned to control levels after culture in vitro (shown in Figure 5A). In vitro culture of SLE T cells with autologous and control normal sera did not prevent the reduction in binding of CTB to GM1 (Figure 6, A and B, upper panels). Also, incubation of $T$ cells from healthy volunteers with SLE sera or autologous serum as controls did not change the level of GM1 expression (Figure 6, $\mathrm{A}$ and $\mathrm{B}$, lower panels). These experiments indicate that SLE serum does not induce the changes in raft-associated GM1 expression observed in T cells from lupus patients. T cells cultured in sera as described above were also analyzed by Western blot for protein tyrosine phosphorylation; however, no differences were observed in the phosphotyrosine profiles of cells incubated in autologous, control, or SLE sera (data not shown).

The expression of LCK was examined by flow cytometry in $\mathrm{T}$ cells isolated ex vivo and in T cells cultured for 72 hours in autologous, control, or SLE sera as described above. LCK expression was reduced in ex vivo T cells from patients with SLE compared with that of controls (Figure 6C). However, culture in autologous, normal, or SLE sera did not prevent LCK levels from recovering to normal levels in SLE T cells, while no change in LCK expression was seen in $\mathrm{T}$ cells from normal controls (Figure 6D). To eliminate the possibility that $\mathrm{T}$ cell apoptosis was obscuring detection of lipid rafts and LCK expression, we assessed the number of cells expressing annexin $\mathrm{V}$ ex vivo and after 72 hours of incubation in the different sera. No differences were revealed between groups (data not shown).

Previous reports have described that $\mathrm{T}$ cells from patients with SLE have reduced proliferative responses to antigen (30). We examined the effect of soluble factors on $\mathrm{T}$ cell function by culturing $\mathrm{T}$ cells for 72 hours in autologous, control, or SLE sera and subsequently stimulating them with antibodies against CD3 and CD28. T cell proliferation was assessed by tritiated thymidine $\left(\left[{ }^{3} \mathrm{H}\right] \mathrm{TdR}\right)$ incorporation. Normal $\mathrm{T}$ cells incubated with sera from SLE patients showed significantly reduced proliferation compared with SLE T cells incubated with sera from healthy controls $(P=0.005)$ (Figure 6E). These results reveal that serum factors alone are not responsible for the alterations in raft-associated GM1 and LCK expression location observed in T cells from patients with SLE and suggest that additional factors in the body are important in initiating $\mathrm{T}$ cell abnormalities in these cells. However, factors from SLE serum downregulate the proliferative capacity of $\mathrm{T}$ cells.

CTB binding and lipid raft occupancy is altered in T cells from SLE patients upon $T$ cell activation. We next explored how altered lipid raft CD45 expression and distribution in lupus $\mathrm{T}$ cells affects their capacity to respond to antigenic triggers. $T$ cells were stimulated in vitro with beads coated with anti-CD3 and anti-CD28 over a time course from 1 to 60 minutes; CTB binding and CD45/lipid raft colocalization were investigated. When CTB binding to GM1 was examined, T cells from patients with SLE had an increased GM1 expression ex vivo, as mentioned earlier; this increase was maintained over a 60-minute activation time course. Condensed areas of raft-associated GM1 expression were seen in the contact site between $T$ cells and activating beads after 1 minute; however, after 15 minutes of activation, rafts were no longer concentrated at the contact zone (Figure 7A, see arrows in upper panels; $\mathrm{X}$ marks the position of the beads coated with anti-CD3 
A
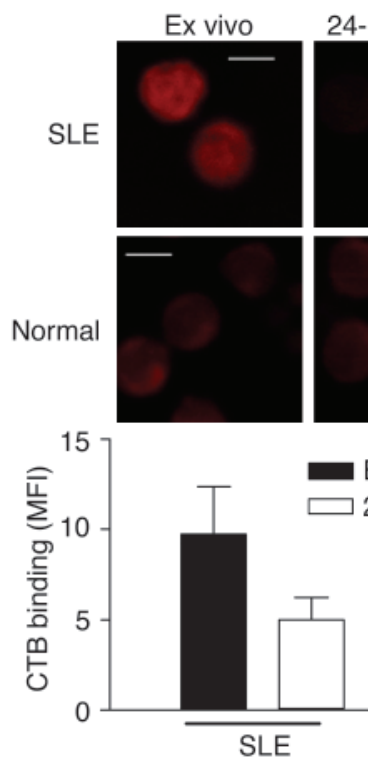

B CD45/CTB overlay
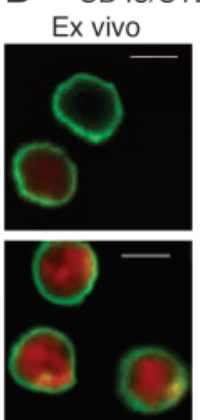
ON "rest"
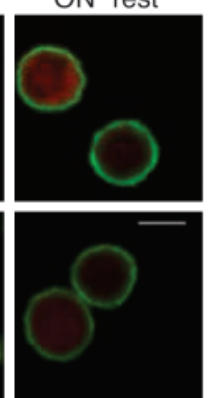

24-hour "rest"

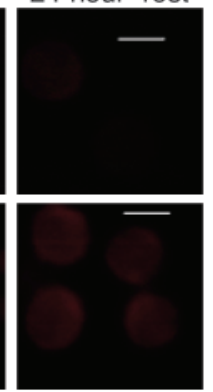

Ex vivo 24-hour "rest"

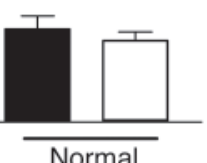

LCK/CTB overlay

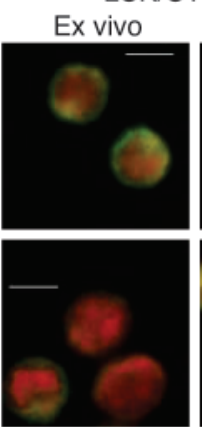

D

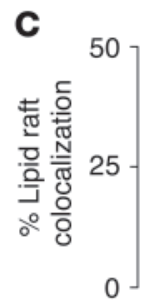

CD45

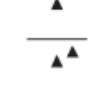

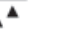

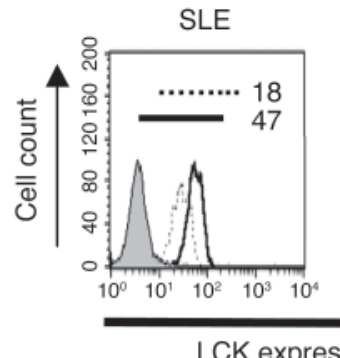

LCK expression in T lymphocytes MFI

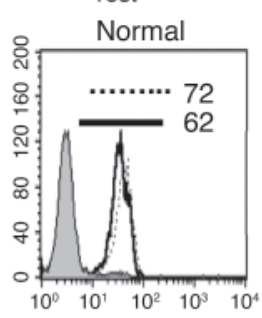

Normal

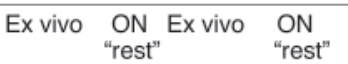

$\triangle$ SLE

$\square$ Normal
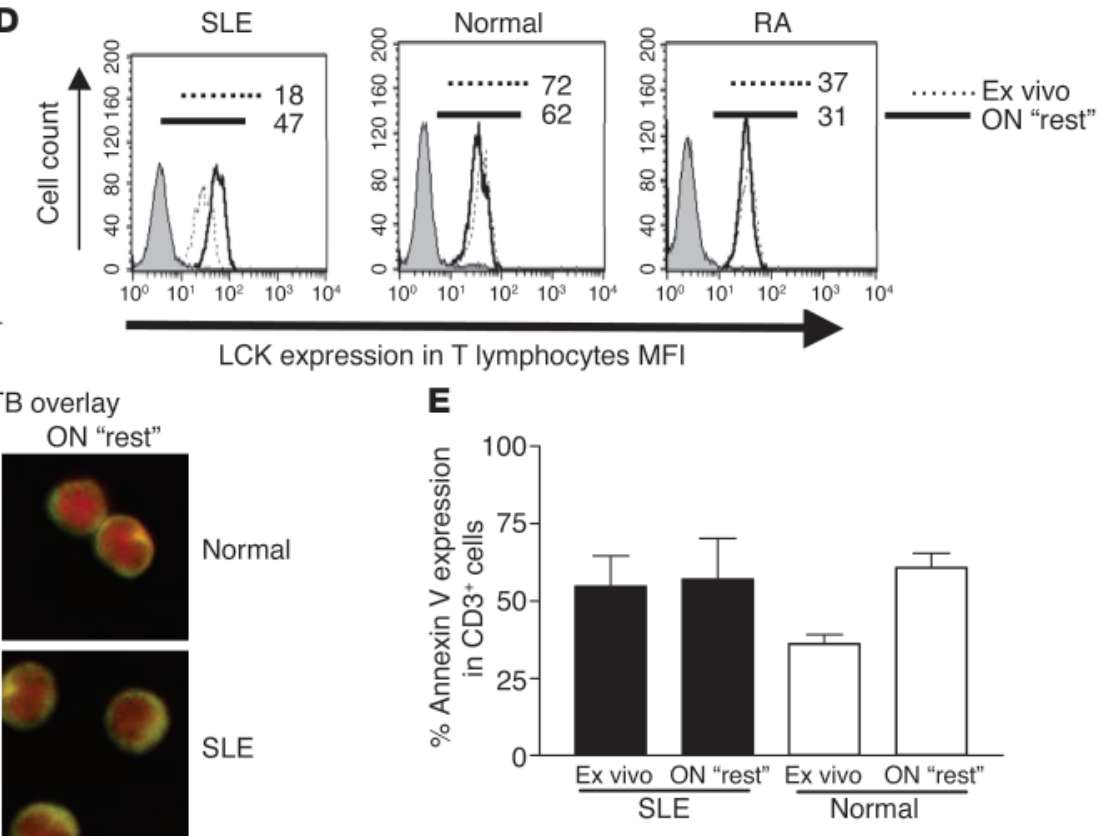

E

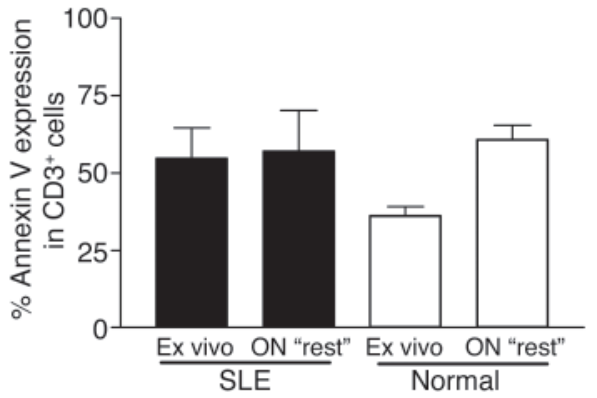

Figure 5

In vitro culture of SLE T cells normalizes expression of GM1 and LCK. T lymphocytes from four patients with SLE and four healthy volunteers (Normal) were analyzed by confocal microscopy either ex vivo or after overnight (ON) "rest." (A) Representative microscopy images showing CTB binding in SLE and normal T cells analyzed ex vivo and after 24 hours of "rest" (upper panels). The CTB binding intensity, measured by MFI ( \pm SEM), in three representative images from three patients with SLE and three healthy controls is shown (lower panel). (B) Representative microscopy images showing CD45/CTB and LCK/CTB colocalization in SLE and normal T cells analyzed ex vivo and after 24 hours of "rest". In $\mathbf{A}$ and $\mathbf{B}$ : scale bars, $5 \mu \mathrm{m}$; X on confocal images represents position of activating bead. (C) Semiquantitative results from four patients with SLE and four healthy controls. On average, 25 cells from each sample were assessed for areas of CD45/CTB or LCK/CTB colocalization. (D) The effect of overnight "rest" on T cell LCK expression was measured by flow cytometry. T cells $\left(1^{6}\right)$ were stained with anti-CD3-PE followed by rabbit anti-LCK or isotype control and FITC-conjugated anti-mouse Ig. Results are expressed as MFI. Figure is representative of three experiments. Numbers indicate MFI. (E) T cell apoptosis was assessed in five patients with SLE and five healthy controls. Cells were labeled with annexin V-FITC and propidium iodide and were analyzed by flow cytometry. Results are expressed as percent of $\mathrm{CD} 3^{+}$cells expressing annexin $\mathrm{V}$ (mean $\pm \mathrm{SEM}$ ).

and anti-CD28). In T cells from healthy controls, GM1 expression was lower than in SLE T cells ex vivo; levels increased after activation to reach a peak at 30 minutes (Figure 7A, lower panels). Lipid raft concentration at the $\mathrm{T}$ cell-bead contact site was observed at a later time point (15 minutes after activation), possibly due to the lower preactivation level of GM1 expression in normal T cells. Additionally, when ex vivo T cells were allowed to "rest" overnight in medium, then were stimulated for 5 minutes with beads coated with antibodies against CD3 and CD28, GM1 expression was rapidly increased in lupus T cells compared with control cells, suggesting that $\mathrm{T}$ cells from patients with SLE are primed for activation (Figure 7B).

The association of CD45 with the T cell-bead contact zone was also examined. After 1 minute of activation, CD45 was rapidly recruited to the contact site in $\mathrm{T}$ cells from both patients with SLE and normal controls (Figure 7C; arrows indicate position of $\mathrm{T}$ cell-beat contact site). Then, in SLE T cells, after 5 minutes of activation, CD45 accumulation was less intense and CD45 was beginning to be excluded from the contact site, while after 10 minutes, CD45 had been excluded completely from the area of contact between $\mathrm{T}$ cell and activation bead (Figure 7C, upper panels). However, in control T cells, CD45 was excluded more slowly; after 15 minutes, CD45 was beginning to be visibly diminished from the $\mathrm{T}$ cell-bead contact zone (Figure 7C, lower panels).

When CD45/lipid raft colocalization was examined, $\mathrm{T}$ cells from patients with SLE showed high levels of CD45/lipid raft association ex vivo; after 1 minute of activation, CD45/lipid raft colocalization was increased and, moreover, areas of colocalization were focused at the points of contact between $\mathrm{T}$ cell and acti- 
A CTB 72-hour incubation

Autologous serum Untreated IgG depleted

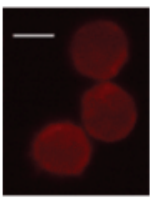

Autologous serum

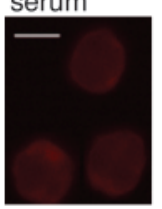

B
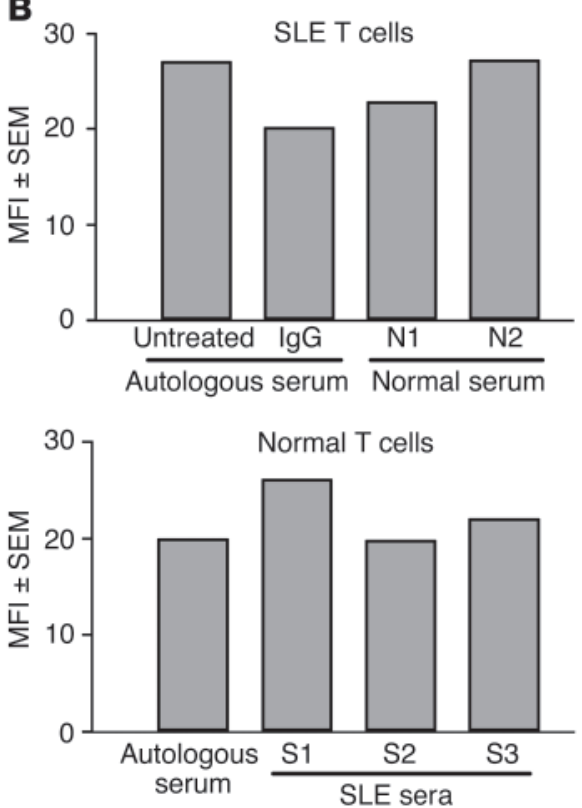

Normal sera

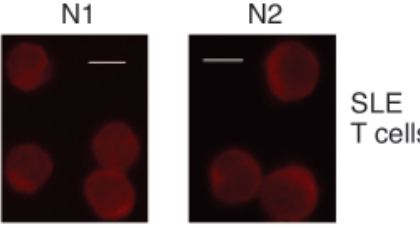

S3

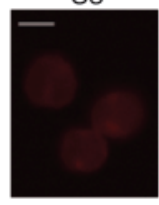

Normal

T cells

\section{D}

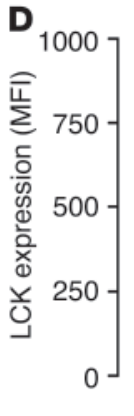

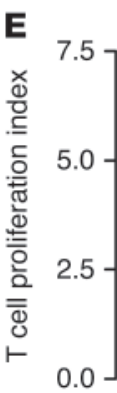
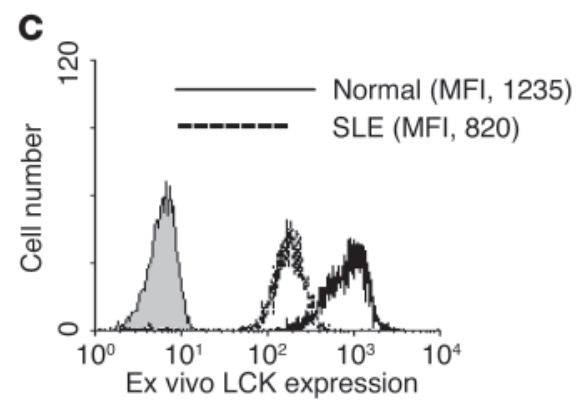

Ex vivo LCK expression

$$
\text { (1) }
$$

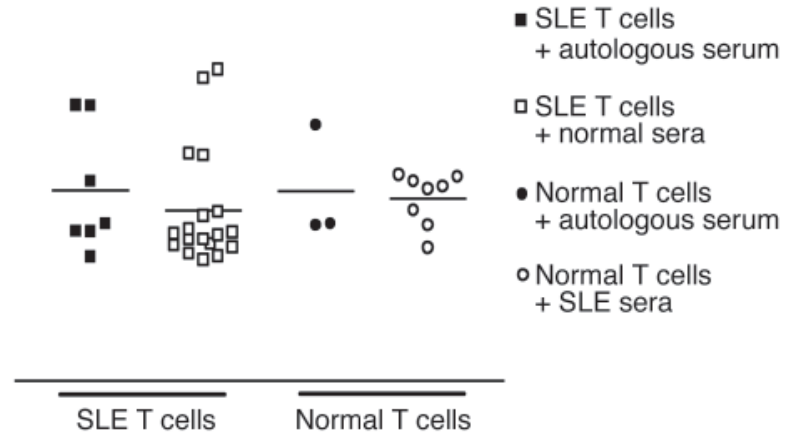

SLE T cells

SLE T cells

Normal T cells

Normal T cells

+ SLE sera

\section{Figure 6}

The effect of soluble serum factors on T cell GM1 and LCK expression and T cell proliferation. T lymphocytes from lupus patients and controls were cultured for 72 hours with $50 \%$ sterile human serum. Some SLE sera were depleted of IgG using protein G-sepharose to remove autoantibodies. Cells were recovered and were fixed for confocal microscopy or were analyzed for LCK expression by flow cytometry. (A) Representative confocal microscopy images showing binding of CTB to GM1 in SLE and normal T cells cultured for 72 hours in autologous, normal, or SLE sera. Scale bars, $5 \mu \mathrm{m}$. N1, N2, sera from two different healthy controls; S1, S2, S3, sera from three different SLE patients. (B) Semiquantitation of CTB binding (MFI) of the images shown in A. (C) Representative experiment showing ex vivo expression of LCK (MFI) in T cells from SLE patients and controls. (D) Cumulative results showing LCK expression (MFI) in SLE and normal T cells after 72 hours in culture with autologous, normal, or SLE sera. (E) T cells recovered from incubation with $50 \%$ human sera were activated for 3 days with anti-CD3 and anti-CD28 and were pulsed with [ $\left.{ }^{3} \mathrm{H}\right] \mathrm{TdR}$. After 18 hours, cells were harvested and $\left[{ }^{3} \mathrm{H}\right] \mathrm{TdR}$ incorporation was measured. Results are shown as a proliferation index (proliferation in autologous serum/proliferation in normal or SLE serum).

vating bead. Over the remaining activation time course, CD45/ lipid raft association resembled ex vivo levels and areas of colocalization were detected at a distance from the $\mathrm{T}$ cell-bead contact zone (Figure 7D, upper panels; areas of colocalization indicated by arrows). Normal $\mathrm{T}$ cells, however, had low levels of CD45/lipid raft colocalization ex vivo, followed by an increase after 1 minute of activation; however, CD45/lipid raft colocalization did not appear to correspond to the site of T cell-bead contact. CD45 was subsequently excluded from raft domains over the activation time course to reach ex vivo levels at 60 minutes (Figure 7D, lower panels).
Furthermore, protein phosphotyrosine profiles mirror lipid raft expression and CD45/lipid raft colocalization (Figure 7E). Activated $\mathrm{T}$ cells were analyzed by Western blot using anti-phosphotyrosine and anti-actin to control for equal loading. SLE T cell protein tyrosine phosphorylation was increased ex vivo in nonstimulated cells, and this tyrosine phosphorylation was increased further after stimulation with beads coated with antiCD 3 and anti-CD28 and remained increased over the activation time course. Normal $T$ cells displayed a peak of protein phosphorylation 5 minutes after activation, which gradually diminished over the 60-minute time course. When we examined the 


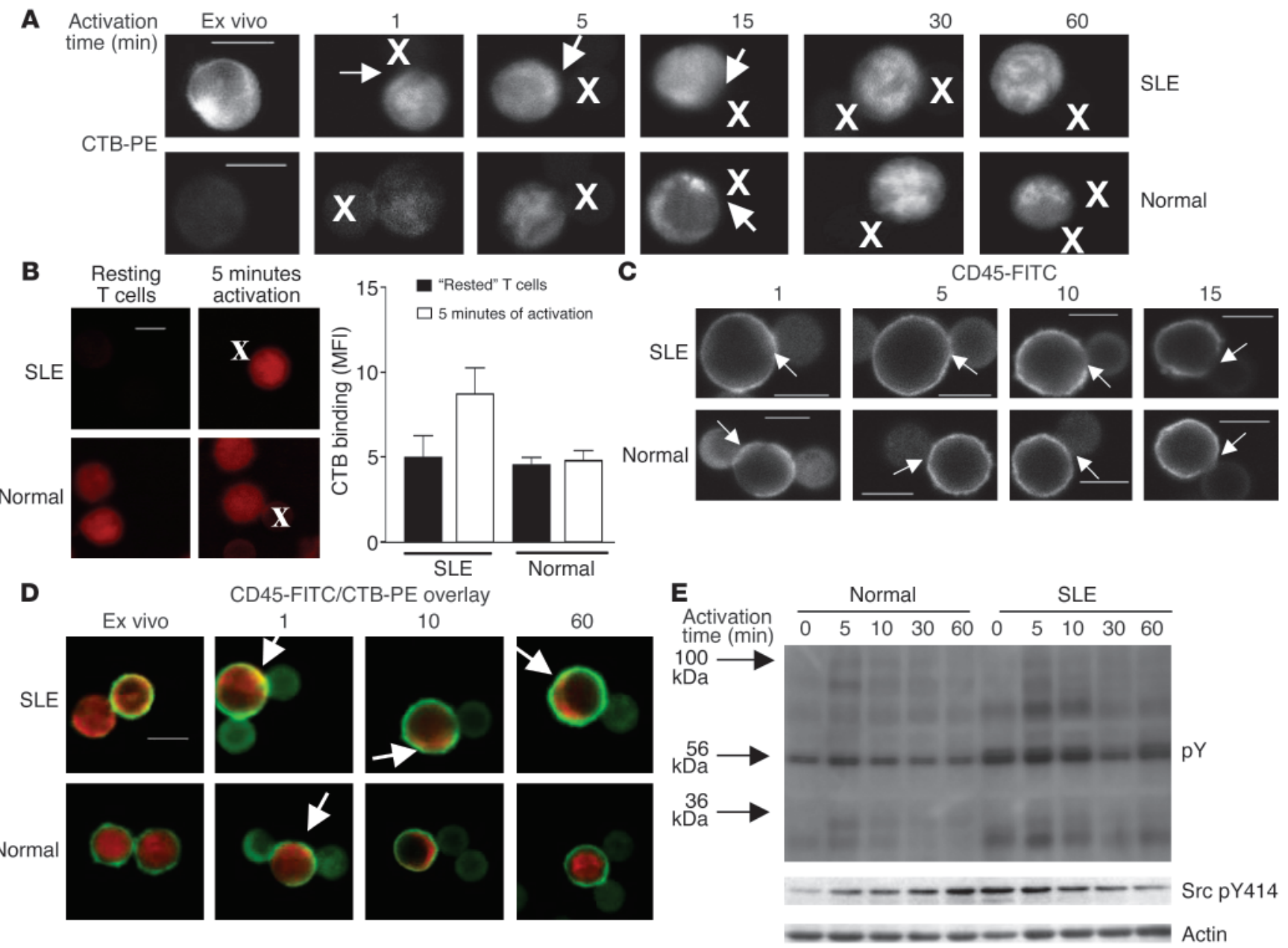

\section{Figure 7}

The effect of T cell activation on GM1 expression and location of CD45. T cells from patients with SLE and healthy controls (Normal) were activated using magnetic beads coated with anti-CD3 and anti-CD28 for 1, 5, 10, 15, 30, and 60 minutes. Activated cells were subsequently fixed for confocal microscopy or lysed for analysis by Western blotting. (A) Figure representative of three experiments showing T cell expression of CTB during TCR activation. (B) T cells from patients with SLE or normal controls were allowed to "rest" overnight in complete medium, then were fixed for confocal microscopy analysis or were activated using magnetic beads coated with anti-CD3 and anti-CD28 for 5 minutes. Activated cells were then fixed for confocal microscopy. The images are representative of four experiments. The bar graph shows semiquantitation of CTB binding (MFI \pm SEM) in five representative images examined from each of four patients with SLE and four healthy controls. (C) Figure representative of three experiments showing T cell expression of CD45 during TCR activation. (D) CD45/CTB overlay images comparing SLE and normal T cells after TCR activation; yellow color indicates areas of colocalization. (E) Proteins from T cell lysates were separated by $8 \%$ SDS-PAGE, transferred to PVDF membranes, and probed with antibodies against phosphotyrosine (pY) and active LCK (Src pY414) and against actin as a control for equal protein loading (Actin). Scale bars, $5 \mu \mathrm{m}$. X on confocal images represents position of activating bead.

expression of the activated form of LCK using antibodies against phospho-Src pY414, an increase in the activated form of LCK was seen in SLE T cells ex vivo (time point 0 ); however, the expression of the "open-active" form of LCK diminished over the activation time course. In normal T cells, low levels of phospho-Src pY414 expression were seen in unstimulated cells, and levels increased gradually after $\mathrm{T}$ cell stimulation.

Taken together, these results indicate that TCR activation induces transient CD45 incorporation to raft domains, as is the case in normal $\mathrm{T}$ cells. However, the kinetics of CD45 distribution into the bead-cell contact area differs. $T$ cells from patients with SLE appear to react more rapidly in terms of lipid raft and CD45 recruitment to the site of TCR activation and CD45 is excluded more rapidly from the activation site; this may be associated with an increase in the proportion of the plasma membrane adopting a lipid raft structure, as determined by GM1 expression in T cells from lupus patients. These results show that $\mathrm{T}$ cells from patients with SLE are primed for activation and respond more rapidly to antigenic triggers than do T cells from normal controls.

\section{Discussion}

Our previous studies have established that LCK expression is reduced in SLE T lymphocytes (26). The experiments described here show that the CD45 tyrosine phosphatase, which regulates LCK activity, is also differentially expressed and its localization into lipid rafts is increased. The results also show higher levels of the glycosphingolipid GM1, a constituent of lipid rafts, in the plasma membrane of SLE T cells. Increased levels of GM1 most 
likely indicate that a higher proportion of the plasma membrane assumes a lipid raft structure. CTB, which specifically binds to GM1, has been used previously to detect lipid rafts $(32,33)$, and accumulated experimental evidence supports the use of GM1 as a credible marker for lipid rafts. Hence, GM1 predominantly copurifies within the lipid raft fraction during biochemical purification (34); cross-linking of CTB-labeled membranes using antibody results in the formation of membrane patches that colocalize with other raft markers, including glycosylphosphatidylinositolanchored receptors and lipidated molecules such as LCK, but not with transferrin receptor, which does not co-purify with lipid rafts; and finally, cholesterol depletion disrupts lipid rafts and CTBstained patches in cells $(6,28)$.

Experiments in the present study addressed the possibility that reduced LCK expression resulted from dysregulated LCK homeostasis in T cells from SLE patients. LCK is maintained in its open primed conformation by interaction with CD45, as described previously $(16,18,28,29)$. Although there was a reduction in the total levels of CD45 expression in unstimulated T cells from patients with SLE (Figure 4A), there was an increase in localization of CD45 to lipid raft domains (Figures 2 and $4 \mathrm{C}$ ) and a parallel increase of activated LCK in these domains (Figure 4C). Additionally, we observed an increase in the association of LCK with CD45 in lupus $\mathrm{T}$ cells compared with that of controls (Figure 4D). The most prominent phospho-proteins in lipid raft domains from unstimulated $\mathrm{T}$ cells are LCK and $\mathrm{Cbp} / \mathrm{PAG}$, an adapter molecule that negatively regulates LCK activity by recruiting CSK (19). Whether CD45 in addition to LCK can also dephosphorylate Cbp/PAG in lipid rafts, leading to dissociation of CSK, is currently unknown. Although our experiments did not reveal alterations in the association of CSK with lipid raft domains, we can not exclude the possibility that association of CD45 with lipid rafts in SLE T cells increases LCK activity not only by dephosphorylating Tyr505 on LCK but also by dephosphorylating Cbp/PAG and thus by disrupting the CSK-Cbp/PAG inhibitory complex.

A body of evidence suggests that CD45 is excluded from lipid raft domains $(16,18,28,29)$. However, recent reports have shown that CD45 is associated with $\mathrm{T}$ cell lipid raft domains in $\mathrm{T}$ cell lines and that it is excluded only after $\mathrm{T}$ cell activation $(35,36)$, thus suggesting that the lipid raft association of CD45 is dependent upon the activation status of the cell. Our results show that $\mathrm{T}$ cells from patients with SLE demonstrate a more pronounced inclusion of CD45 to raft domains and that CD45 is maintained in these domains even after stimulation of the TCR (Figure 7). These results point to the possibility that alterations in the distribution of CD45 in T cells from patients with SLE may contribute to abnormalities in these cells.

Additionally, compared with those from healthy controls, T cells from patients with SLE react more rapidly in terms of lipid raft and CD45 recruitment to the site of TCR activation. After its initial recruitment, CD45 is excluded more rapidly from the cell-bead contact site in SLE T cells, although it is still associated with raft domains away from the TCR activation region (Figure 7C). We assume that if LCK levels remain above a critical threshold for signaling, then higher LCK activity may result in hyper-responsive $\mathrm{T}$ cells primed to respond to TCR/CD28 stimulation more readily. This is confirmed in Figure 7E, in which increased levels of active LCK correspond with increased protein tyrosine phosphorylation after $T$ cell activation in SLE. These results could suggest that $T$ cells from patients with SLE are primed for activation and respond more rapidly to antigenic triggers than do T cells from normal controls and provide an explanation for the exaggerated response to environmental antigens observed in patients with SLE (37).

Increased LCK ubiquitination and subsequent degradation seen in SLE T cells (26) could result from increased consumption of the activated form of LCK, possibly due to the altered localization of CD45 and its increased interaction with LCK (Figures 2, C and D, and 4). It was recently demonstrated that ubiquitin-mediated degradation of active LCK is controlled by the c-Cbl ubiquitin ligase (38). In turn, c-Cbl is independently ubiquitinated and degraded during this process (39). Interestingly, we observed reduced expression of $\mathrm{c}-\mathrm{Cbl}$ in lupus $\mathrm{T}$ cells (Figures $3 \mathrm{~A}$ and $4 \mathrm{D}$ ), supporting the results of a recent report by Datta and colleagues (40) that demonstrated abnormalities in Cbl-mediated regulation of signaling pathways in $\mathrm{T}$ cell lines derived from patients with SLE. However, we cannot exclude the possibility that the antibody used to detect c-Cbl may also cross-react with the homologous molecule Cbl-b, therefore indicating possible reduced expression of this molecule as well.bCbl-b is shown be critical in regulating the threshold for $\mathrm{T}$ cell activation; deficiency in Cbl-b uncouples peripheral $\mathrm{T}$ cell proliferation from CD28 costimulation, resulting in lupus-like autoimmunity in mouse models (41-43). Furthermore, Cbl-b was shown to negatively regulate aggregation of rafts in T cells (44). Thus, the reduction in Cbl molecules may have profound consequences on $\mathrm{T}$ cell activation threshold in lupus T cells.

Interestingly, when purified SLE T cells were allowed to "rest" in vitro, the changes seen in LCK, CD45, and lipid raft expression in freshly purified cells were reversed. Addition of serum from patients with SLE during the in vitro incubation did not prevent this reversion of cell phenotype, suggesting that factors present in SLE serum are not sufficient for reproducing the SLE phenotype in vitro. It is possible that while in the body of the patient, T cells come into contact with other cell types and this interaction is responsible, at least in part, for the observed alterations in the proximal signaling pathways. Relevant to this hypothesis is a recent report by Blanco and colleagues (31), which demonstrated that $\mathrm{CD} 14^{+}$monocytes differentiate into plasmacytoid dendritic cells under the influence of SLE serum. These results revealed that IFN- $\alpha$, whose levels are increased in patients with SLE (45), is a factor driving dendritic cell differentiation and activation in SLE. An increase in the differentiation of plasmacytoid dendritic cells, resulting in increased presentation of autoantigens to $\mathrm{T}$ and $\mathrm{B}$ lymphocytes, could lower the threshold for $\mathrm{T}$ and $\mathrm{B}$ cell activation. Hence, we found that when lupus $\mathrm{T}$ cells were allowed to "rest" in vitro, lipid rafts were reduced but they were rapidly overexpressed following subsequent activation with anti-CD3 and anti-CD28.

Upregulation of CTB binding to GM1 most likely indicates an increase in lipid raft formation in lupus $\mathrm{T}$ cells and could be linked to $\mathrm{T}$ cell phenotype and differential association of signaling molecules with raft domains. An increase in lipid raft formation is likely to increase the strength of the $T$ cell signal, reducing the threshold for $\mathrm{T}$ cell stimulation. This in turn may influence immune cell function in SLE patients, and thus lipid rafts may provide targets for future therapy (46). Lipid raft heterogeneity, in terms of both protein and lipid content, has been described in relation to $\mathrm{T}$ cell activation and function $(33,47-50)$. Current evidence supports an "induced fit" model of raft heterogeneity wherein individual raft proteins dynamically interact with raft lipids, inducing a constant remodeling of the raft constituents, serving to produce unique rafts tailored to the structure of the protein and lipid con- 
stituents (49). Thus, decreased lateral mobility of T cell surface receptors and reduced capping in $\mathrm{T}$ cells from patients with SLE may reflect abnormalities in lipid raft composition (51). Increased lipid raft expression has also been reported previously in activated and memory $T$ cells compared with naive $T$ cells $(32,33)$. Thus, the increased raft expression in SLE T cells could be indicative of an "activated" phenotype that could result from the exposure of $\mathrm{T}$ cells to differentiated dendritic cells expressing relevant coreceptors, cytokines, circulating immune complexes, or a combination of these factors. Alternatively, the increased expression of GM1 and therefore CTB binding may result from altered activity of lipid targeting enzymes such as sialidase or endoglycanase. However, to our knowledge, there is no evidence that in SLE T cells such enzymes have differential activity or that the TCR or other costimulatory receptors can regulate the activity of endoglycanase enzymes.

Our results corroborate studies by Tsokos and colleagues suggesting that T cells from patients with SLE are "rewired" and signal using alternative pathways (52), including signaling via the Fc receptor $\gamma$ chain, which, although not present in normal $\mathrm{T}$ cells, is expressed in T cells from patients with SLE (27). Thus, anomalous expression and changes in membrane location of signaling molecules could contribute to the alteration in the threshold for $\mathrm{T}$ cell activation in patients with SLE.

In conclusion, we have shown here that reduced expression of LCK in T cells from patients with SLE is associated with increased expression of the raft-associated glycoshpingolipid GM1, most likely indicating increased formation of raft structures and increased raft localization of CD45, a molecule important in regulating LCK activity. We have also observed that alterations in lipid raft expression and occupancy can be reversed by resting $T$ cells ex vivo but are induced again by TCR activation, suggesting that SLE T cells are primed to respond rapidly to such stimulation. It remains to be established whether these defects are intrinsic abnormalities or result from the effect of other factors such as high levels of cytokines or abnormal $\mathrm{T}$ cell costimulation. The answer to these questions may lead to the development of novel therapeutic interventions to correct the abnormalities in $\mathrm{T}$ cell function in these patients.

\section{Methods}

Patients and controls. Nineteen SLE patients fulfilling the American College of Rheumatology revised classification criteria for lupus were included in this study (mean age, 38 years; range 16-66 years; sixteen female and three male) (53). Disease activity in the SLE patients was assessed using the British Isles Lupus Assessment Group (BILAG) index (54). Patients with a BILAG global score of more than 6 were considered to have active disease; those with a score of 5 or below were considered to have inactive lupus. In our cohort, ten patients had active disease and nine had inactive disease. Treatment groups of SLE patients were classified as follows: A, no treatment, nonsteroidal anti-inflammatory drugs, anti-malarial drugs, and/or low-dose steroids $(<10$ $\mathrm{mg} /$ day); and $\mathrm{B}$, high-dose steroids (>10 mg/day) and/or immunosuppressive therapy. Seven patents were in treatment group A and twelve patients were in treatment group B. Fifteen healthy individuals (mean age, 34 years; range, 23-50 years; two male and thirteen female) and six patients with RA (mean age, 52 years; range, 22-81 years; four female and two male) were studied in parallel as controls; two RA patients were in treatment group A and four RA patients were in treatment group B. The ethics committee of the University College London Hospitals National Health Service Trust approved the study; patients and healthy volunteers were recruited after informed consent was obtained. Antibodies. Antibodies against human CD19 (RFB9), CD14 (AML), CD56 (B159) and CD16 (3G8) were purified from mouse hybridoma cell lines (Chemicon, Southampton, UK). Purified mouse monoclonal antibody (mAb) against LCK (3A5), 3A5 conjugated to sepharose, and CD45 (35-Z6), and rabbit polyclonal antibodies against LCK, c-Cbl, and CSK were obtained from Santa Cruz Biotechnology (Santa Cruz, California, USA). Rabbit polyclonal antibodies against the active (phospho-Src pY414) and inactive (phospho-LCK pY505) forms of LCK were obtained from New England Biolabs (Hertfordshire, United Kingdom). Antibodies against phospho-Src pY414 are cross-reactive with other Src family kinases including LCK; this antibody was used because specific anti-phospho-LCK pY394 was not available. Mouse mAb against phosphotyrosine (4G10) and rabbit polyclonal antibody against LAT were obtained from Upstate Biotechnologies (Lake Placid, New York, USA). Purified mAb against CD45 (H130) and conjugated antibodies to human CD3-FITC, CD45-PE, CD25-PE, CD69$\mathrm{PE}$, and isotype controls were obtained from $\mathrm{BD}$ Bioscience (Oxford, United Kingdom), whereas antibodies against human CD3-PE, CD45RA-PE, and CD45RO-PE, and CD3-PE-indodicarbocyanine (CD3-PE-Cy5)/CD4-PE/CD8-FITC were obtained from DAKO (Carpinteria, California, USA). Rabbit Ig control serum, rabbit polyclonal antibody to actin together with rabbit anti-mouse and goat anti-rabbit HRP- and FITC-conjugated antibodies were obtained from Sigma-Aldrich (Dorset, United Kingdom).

Isolation and culture of Tlymphocytes. Peripheral blood mononuclear cells were isolated from approximately $40 \mathrm{ml}$ of heparinized venous blood from patients with SLE or controls by centrifugation over Ficoll-Hypaque (Pharmacia Biotech, Buckinghamshire, United Kingdom). T lymphocyte-enriched populations were obtained by negative selection using mouse mAb against human CD14, CD16, CD56, and CD19 $\left(0.5 \mu \mathrm{g} / 10^{6}\right.$ cells $)$ and magnetic beads coated with anti-mouse Ig (Dynal, Oslo, Norway) with a method described previously (26). For overnight cell culture, T lymphocytes from lupus patients and controls were cultured at $37^{\circ} \mathrm{C}$ in $5 \% \mathrm{CO}_{2}$ for 18 hours in complete RPMI 1640 medium (Life Technologies, Paisley, United Kingdom) with $10 \%$ fetal calf serum (Life Technologies), $100 \mathrm{U} / \mathrm{ml}$ penicillin, $50 \mu \mathrm{g} / \mathrm{ml}$ streptomycin, and $1 \mathrm{mM}$ amphotericin (SigmaAldrich). Cells were recovered and were fixed for confocal microscopy or were activated using magnetic beads coated with anti-CD3 and anti-CD28 (Dynal) for 1, 5, 10, 15, 30, and 60 minutes at $37^{\circ} \mathrm{C}$. Activated cells were fixed for confocal microscopy analysis. For serum incubation experiments, T cells were incubated for 72 hours at $37^{\circ} \mathrm{C}$ in 5\% $\mathrm{CO}_{2}$ with RPMI 1640 medium and 50\% sterile human serum. SLE sera were depleted of IgG using protein G-sepharose (a suspension of equal volumes of serum and sepharose) (Pharmacia Biotech) mixed for 2 hours at $4{ }^{\circ} \mathrm{C}$. Sera were checked for antibody depletion using an anti-human IgG ELISA (55). For proliferation experiments, $\mathrm{T}$ cells recovered from incubation with $50 \%$ human sera were plated in 96-well plates ( $10^{5}$ cells/well in complete RPMI medium) precoated with anti-CD3 $(10 \mu \mathrm{g} / \mathrm{ml})$ and with soluble anti-CD28 $(10 \mu \mathrm{g} / \mathrm{ml})$ added to the cultures. Cells were incubated for 3 days and were pulsed with $1 \mu \mathrm{Ci} / \mathrm{ml}\left[{ }^{3} \mathrm{H}\right] \mathrm{TdR}$ for the last 18 hours. Cell proliferation was determined by $\left[{ }^{3} \mathrm{H}\right] \mathrm{TdR}$ incorporation.

Flow cytometry. The purity of T lymphocytes in the enriched populations was determined by flow cytometry using anti-CD45-PE and anti-CD3-FITC and appropriate isotype controls and was 
consistently greater than $94 \%$. T lymphocyte phenotype was analyzed further using triple staining for CD3-PE-Cy5/CD4-PE/CD8FITC and activation status was assessed using anti-CD25-PE, anti-CD69-PE, anti-CD45RA-PE, and anti-CD45RO-PE. Cells were fixed in $2 \%$ paraformaldehyde and were analyzed by flow cytometry using a FACSan with CellQuest software (BD Bioscience). $\mathrm{T}$ cell apoptosis was assessed by labeling with annexin $\mathrm{V}$ (FITC) and propidium iodide according to manufacturer's instructions (BD Bioscience).

Intracellular LCK staining was according to an established protocol with minor modifications (56). Cells $\left(10^{6}\right)$ were stained with anti-CD3-PE for 20 minutes in staining buffer (SB) (PBS, 2\% FCS, and $0.1 \%$ sodium azide) and were fixed in $2 \%$ paraformaldehyde. After being washed in SB, cells were incubated with $0.3 \%$ saponin (Sigma-Aldrich) in SB (SB-Sap) for 10 minutes at room temperature. Cells were incubated with rabbit anti-LCK (1:100 dilution) in SB-Sap for a further 20 minutes. After being washed in SB-Sap, cells were incubated with anti-rabbit IgG-FITC in SB-Sap for 20 minutes followed by two washes in SB-Sap and one wash in SB. Cells were analyzed as described above.

Confocal microscopy. T cells were incubated in RPMI 1640 for 15 minutes at $37^{\circ} \mathrm{C}$, washed in sterile PBS, fixed in $4 \%$ paraformaldehyde, and washed again before being applied to washed multiwell microscope slides $\left(2 \times 10^{5}\right.$ cells per well) (Hendly, Essex, United Kingdom) coated with $0.1 \%$ 3-aminopropyltriethoxy silane (TESPA; Sigma-Aldrich). Attached cells were incubated for $10 \mathrm{~min}$ utes with blocking buffer with $0.3 \%$ saponin to make the cells permeable (BB-Sap: PBS with $0.5 \%$ fish gelatin [Sigma-Aldrich] and $0.3 \%$ saponin) followed by primary antibody diluted in BB-Sap (polyclonal anti-LCK, anti-CSK, or anti-c-Cbl at a dilution of 1:100 or anti-CD4 at a dilution of 1:300) for 60 minutes, then were washed three times in BB-Sap. Secondary antibody diluted in BBSap (anti-rabbit Ig-FITC or anti-mouse Ig-FITC; 1:100 dilution) was applied for 30 minutes at room temperature with protection from light. After three washes, cells were stained for lipid rafts by incubation with CTB-PE conjugate (1:100 dilution) (Molecular Probes Inc., Eugene, Oregon, USA) in blocking buffer for 15 minutes. Finally, cells were washed and slides were covered with coverslips using mowiol solution (Calbiochem, San Diego, California, USA). Cells were analyzed with a Bio-Rad MRC 1024 confocal system (Bio Rad Laboratories, Hertfordshire, United Kingdom) equipped with an argon and helium/neon laser for excitation at $522 \mathrm{~nm}$ and $585 \mathrm{~nm}$. Images were acquired with LaserSharp 3.2 software. FITC or PE fluorescence was recorded sequentially using a $40 \times$ objective. For overlays, images were adjusted to the same output intensities and merged into a composite image using Confocal Assistant 4.2 software. Images were analyzed for areas of colocalization (yellow color). The results presented are based on analysis of an average of 50 cells from each sample. The quantitative analysis of lipid raft expression was made by measurement of CTB-PE MFI in individual images using Confocal Assistant 4.2 software.
Preparation of lipid rafts. T cells were mixed with $500 \mu \mathrm{l}$ lysis buffer (1\% Triton-X100 in 2-N-morpholino ethanesulfonic acid, sodium chloride, EDTA [MNE] buffer, $25 \mathrm{mM}$ 2- $\mathrm{N}$-morpholino ethanesulfonic acid [MES], pH 6.5, 2 mM EDTA, and $150 \mathrm{mM} \mathrm{NaCl}$ ) with phosphatase inhibitors ( $1 \mathrm{mM}$ sodium orthovanadate and $10 \mathrm{mM}$ sodium fluoride) (Sigma-Aldrich) and protease inhibitors $(500 \mu \mathrm{M}$ 4-[2-aminoethyl0-benzenesulfonyl fluoride] $\mathrm{HCl}$ [AEBSF], $150 \mathrm{nM}$

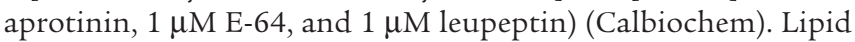
raft and non-raft membrane fractions were isolated by sucrose gradient ultracentrifugation as described previously (26). The resulting raft and non-raft fractions were stored at $80^{\circ} \mathrm{C}$ until analysis.

Western blot and immunoprecipitation. Raft and non-raft fractions were separated under reducing conditions on $10 \%$ or $8 \%$ polyacrylamide gels. For LCK immunoprecipitation, equal quantities of protein from $\mathrm{T}$ cell lysates $(50 \mu \mathrm{g})$ were precleared by rotation at $4{ }^{\circ} \mathrm{C}$ for 30 minutes with $40 \mu \mathrm{l}$ of $50 \%$ packed protein $\mathrm{G}$-sepharose beads (Pharmacia Biotech). Precleared lysates were incubated overnight at $4^{\circ} \mathrm{C}$ with LCK-conjugated protein $\mathrm{G}$-sepharose beads. The beads were washed three times in lysis buffer and pellets were resuspended in Laemmli's sample buffer prior to separation by $8 \%$ SDS-PAGE. Proteins were transferred to PVDF membranes (Millipore) and were quenched for 1 hour at room temperature with blocking buffer containing either $10 \%$ bovine serum albumin or $5 \%$ nonfat milk in PBS plus $0.1 \%$ Tween20 . The membranes were incubated with primary antibody (antibodies against CD 45, LCK, CSK, and c-Cbl $[1 \mu \mathrm{g} / \mathrm{ml}])$ followed by the appropriate HRP-conjugated secondary antibody (1:20,000 dilution) before detection using ECL (Pharmacia Biotech). For subsequent reprobing, membranes were stripped of bound antibodies with stripping buffer $(62.5 \mathrm{mM}$ Tris- $\mathrm{HCl}, 2 \% \mathrm{SDS}$, and 100 $\mathrm{mM} 2$-mercaptoethanol, $\mathrm{pH} 6.7$ ) at $56^{\circ} \mathrm{C}$ for 20 minutes. ECL film was analyzed using the AlphaImager System.

Statistical analysis. Statistical analyses were made using the MannWhitney $U$ test and Kruskall-Wallis tests (nonparametric) or Student's $t$ test (parametric) to compare between sample groups. Statistical significance of the data was set at $P<0.05$.

\section{Acknowledgments}

Lupus UK and Sir Simon Scott of Yews Research Fund sponsored this work. P.S. Kabouridis is supported by a Wellcome Trust Career Development Award (058408). The Arthritis Research Campaign supports F. Flores-Borja, R.A. Mageed, and D.A. Isenberg.

Received for publication October 20, 2003, and accepted in revised form February 17, 2004.

Address correspondence to: Elizabeth C. Jury, Centre for Rheumatology, University College London, Windeyer Institute of Medical Science, 46 Cleveland Street, London W1P 4JF, United Kingdom. Phone: 44-0-207-679-9634; Fax: 44-0-207-679-9143; E-mail: e.jury@ucl.ac.uk.

\footnotetext{
1. Morrow, J., Nelson, L., Watts, R., and Isenberg, D. 1999. Systemic lupus erythematosus. In Autoimmune rheumatic disease. 2 nd edition. Oxford University Press. Oxford, United Kingdom. 56-103.

2. Dayal, A.K., and Kammer, G.M. 1996. The T cell enigma in lupus. Arthritis Rheum. 39:23-33.

3. Tsokos, G.C., and Liossis, S.N. 1999. Immune cell signalling defects in lupus: activation, anergy and death. Immunol. Today. 20:119-124.

4. Simons, K., and Ikonen, E. 1997. Functional rafts in
}

cell membranes. Nature. 387:569-572.

5. Simons, K., and Toomre, D. 2000. Lipid rafts and signal transduction. Nat. Rev. Mol. Cell Biol. 1:31-39.

6. Janes, P.W., Ley, S.C., Magee, A.I., and Kabouridis, P.S. 2000. The role of lipid rafts in T cell antigen receptor (TCR) signalling. Semin. Immunol. 12:23-34.

7. Kabouridis, P.S., Magee, A.I., and Ley, S.C. 1997. $\mathrm{S}$-acylation of LCK protein tyrosine is essential for its signalling function in T lymphocytes. $E M B O J$. 16:4983-4998.
8. Zhang, W., Trible, R.P., and Samelson, L.E. 1998. LAT palmitoylation: its essential role in membrane microdomain targeting and tyrosine phosphorylation during $T$ cell activation. Immunity. 9:239-246.

9. Xavier, R., Brennan, T., Li, Q., McCormack, C., and Seed, B. 1998. Membrane compartmentation is required for efficient $\mathrm{T}$ cell activation. Immunity. 8:723-732.

10. Montixi, C., et al. 1998. Engagement of T cell receptor triggers its recruitment to low-density 
detergent-insoluble membrane domains. EMBO J. 17:5334-5348.

11. Kabouridis, P.S., Janzen, J., Magee, A.L., and Ley, S.C. 2000. Cholesterol depletion disrupts lipid rafts and modulates the activity of multiple signaling pathways in T lymphocytes. Eur. J. Immunol. 30:954-963.

12. Gringhuis, S.I., et al. 2000. Displacement of linker for activation of $\mathrm{T}$ cells from the plasma membrane due to redox balance alterations results in hyporesponsiveness of synovial fluid lymphocytes in rheumatoid arthritis. J. Immunol.164:2170-2179.

13. Salojin, K.V., Zhang, J., Madrenas, J., and Delovitch, T.L. 1998. T-cell anergy and altered T-cell receptor signaling: effects on autoimmune disease. Immunol. Today. 19:468-473.

14. Chan, A.C., Desai, D.M., and Weiss, A. 1994. The role of protein tyrosine kinases and protein tyrosine phosphatases in $\mathrm{T}$ cell antigen receptor signal transduction. Annu. Rev. Immunol. 12:555-592.

15. Williams, J.C., Wierenga, R.K., and Saraste, M. 1998. Insights into Src kinase functions-structural comparisons. Trends Biochem. Sci. 23:179-184.

16. Hermiston, M.L., Xu, Z., Majeti, R., and Weiss, A 2002. Reciprocal regulation of lymphocyte activation by tyrosine kinases and phosphatases. J. Clin. Invest. 109:9-14. doi:10.1172/JCI200214794.

17. Mustelin, T., and Tasken, K. 2003. Positive and negative regulation of $\mathrm{T}$-cell activation through kinases and phosphotases. Biochem. J. 371:15-27.

18. Hermiston, M.L., Xu, Z., and Weiss, A. 2003. CD45: A critical regulator of signalling thresholds in immune cells. Annu. Rev. Immunol. 21:107-137.

19. Brdicka, T., et al. 2000. Phosphoprotein associated with glycosphingolipid-enriched microdomains (PAG), a novel ubiquitously expressed transmembrane adaptor protein, binds the protein tyrosine kinase csk and is involved in regulation of $\mathrm{T}$ cell activation. J. Exp. Med. 191:1591-1604.

20. Kawabuchi, M., et al. 2000. Transmembrane phosphoprotein Cbp regulates the activities of Src-family tyrosine kinases. Nature. 404:999-1003.

21. Kabouridis, P.S. 2003. Selective interaction of LAT (linker of activated $\mathrm{T}$ cells) with the open-active form of Lck in lipid rafts reveals a new mechanism for the regulation of Lck in T cells. Biochem. J. 371:907-915.

22. Latour, S., and Veillette, A. 2001. Proximal protein tyrosine kinases in immunoreceptor signalling. Curr. Opin. Immunol. 13:299-306.

23. Rao, N., et al. 2002. Negative regulation of Lck by Cbl ubiquitin ligase. Proc. Natl. Acad. Sci. U. S. A. 99:3794-3799.

24. Hawash, I.Y., Kesavan, K.P., Magee, A.I., Geahlen, R.L., and Harrison, M.L. 2002. The Lck SH3 domain negatively regulates localisation to lipid rafts through an interation with c-Cbl.J. Biol. Chem. 277:5683-5691.

25. Krawczyk, C., and Penninger, J.M. 2001. Molecular controls of antigen receptor clustering and autoimmunity. Trends Cell Biol. 11:212-220.

26. Jury, E.C., Kabouridis, P.S., Abba, A., Mageed, M.A., and Isenberg, D.A. 2003. Increased ubiquitination and reduced expression of LCK in T lymphocytes from patients with systemic lupus erythematosus. Arthritis Rheum. 48:1343-1354.

27. Nambiar, M.P., et al. 2002. Abnormal expression of various molecular forms and disruption of $\mathrm{T}$ cell receptor $\zeta$ chain in patients with systemic lupus erythematosus. Arthritis Rheum. 46:163-174.

28. Janes, P.W., Ley, S.C., and Magee, A.I. 1999. Aggregation of lipid rafts accompanies signaling via the T cell antigen receptor. J. Cell Biol.174:447-461.

29. Rodgers, W., and Rose, J.K. 1996. Exclusion of CD45 inhibits activity of p56lck associated with glycolipid-enriched membrane domains. J. Cell Biol. 135:1515-1523.

30. Horowitz, D.A., Stohl, W., and Gray, J.D. 2002. T lymphocytes, natural killer cells and immune regulation. In Dubois' lupus erythematosus. 6th edition. D.J. Wallace and B.H. Hann, editors. Lippincott, Williams and Wilkins. Philadelphia, Pennsylvania, USA. 157-185

31. Blanco, P., Palucka, A.K., Gill, M., Pascual, V., and Banchereau, J. 2001. Induction of dendritic cell differentiation by IFN- $\alpha$ in systemic lupus erythematosus. Science. 294:1540-1543.

32. Viola, A., Schroeder, S., Sakakibara, Y., and Lanzavecchia, A. 1999. T lymphocyte costimulation mediated by reorganisation of membrane microdomains. Science. 283:680-682.

33. Tuosto, L., et al. 2001. Organisation of plasma membrane functional rafts upon $\mathrm{T}$ cell activation. Eur. J. Immunol. 31:345-349.

34. Brown, D.A., and London, E. 1998. Functions of lipid rafts in biological membranes. Annu. Rev. Cell. Dev. Biol. 14:111-136

35. Edmonds, S.D., and Ostergaard, H.L. 2002. Dynamic association of CD45 with detergent-insoluble microdomains in $\mathrm{T}$ lymphocytes. J. Immunol. 169:5036-5042.

36. Irles, C., et al. 2003. CD45 ectodomain controls interaction with GEMs and Lck activity for optimal TCR signaling. Nat. Immunol. 4:189-197.

37. Dar, O., Salamam, M.R., Seifert, M.H., and Isenberg, D.A. 1990. Spontaneous antibody secreting cells against DNA and common environmental antigens in systemic lupus erythematosus. J. Autoimmunity. 3:523-530.

38. Yokouchi, M., et al. 2001. Src-catalysed phosphorylation of c-Cbl leads to the interdependent ubiquitination of both proteins. J. Biol. Chem. 276:35185-35193.

39. Yinon, B.-N. 2002. Regulatory functions of ubiquitination in the immune system. Nat. Immunol. 3:20-26

40. Yi, Y., McNerney, M., and Datta, S.K. 2000. Regulatory defects in $\mathrm{Cbl}$ and mitogen-activated protein kinase (extracellular signal-related kinase) pathways cause persistent hyperexpression of CD40 ligand in human lupus T cells. J. Immunol. 165:6627-6634.

41. Bachmaier, K., et al. 2000. Negative regulation of lymphocyte activation and autoimmunity by the molecular adaptor Cbl-b. Nature. 403:211-216.

42. Chiang, Y.J., et al. 2000. Cbl-b regulates the CD28 dependence of T-cell activation. Nature. 403:216-220.

43. Liu, Y.-C., and Gu, H. 2002. Cbl and Cbl-b in T-cell regulation. Trends Immunol. 23:140-143.

44. Krawczyk, C., et al. 2000. Cbl-b is a negative regulator of receptor clustering and raft aggregation in $\mathrm{T}$ cells. Immunity. 13:463-473.

45. Hooks, J.J., et al. 1979. Immune interferon in the circulation of patients with autoimmune disease. N. Engl. J. Med. 301:5-8.

46. Dykstra, M., et al. 2003. Location is everything: Lipid rafts and immune cell signalling. Annu. Rev. Immunol. 21:457-481.

47. Schade, A.E., and Levine, A.D. 2002. Lipid raft heterogeneity in human peripheral blood $\mathrm{T}$ lymphoblasts: A mechanism for regulating the initiation of TCR signal transduction. J. Immunol. 168:2233-2239.

48. Drevot, P., et al. 2002. TCR signal initiation machinery is pre-assembled and activated in a subset of membrane rafts. EMBO J. 21:1899-1908.

49. Pike, L.J. 2004. Lipid rafts: Heterogeneity on the high seas. Biochem. J. 378:281-292.

50. Gomez-Mouton, C., et al. 2001. Segregation of leading edge and uropod components into specific lipid rafts during T cell polarization. Proc. Natl. Acad. Sci. U. S. A. 98:9642-9647.

51. Kammer, G.M. 1983. Impaired T cell capping and receptor regeneration in active systemic lupus erythematosus: evidence for a disorder intrinsic to the T lymphocyte. J. Clin. Invest. 72:1686-1697.

52. Tsokos, G.C., Nambiar, M.P., Tenbrock, K., and Juang, Y-T. 2003. Rewiring the T-cell: signalling defects and novel prospects or the treatment of SLE. Trends Immunol. 24:259-263.

53. Tan, E.M., et al. 1982. The 1982 revised criteria for the classification of systemic lupus erythematosus. Arthritis Rheum. 22:1271-1277.

54. Hay, E.M., et al. 1993. The BILAG index: a reliable and valid instrument foe measuring clinical disease activity in systemic lupus erythematosus. Q. J. Med. 86:447-458.

55. Mason, L.J., Ravirajan, C.T., Latchman, D.S., and Isenberg, D.A. 2001. A human anti-dsDNA monoclonal antibody causes hyaline thrombi formation in kidneys of 'leaky' SCID mice. Clin. Exp. Immunol. 126:137-142.

56. Trowbridge, P.A., and Levin, S.D. 2001. Lck plays a critical role in $\mathrm{Ca}^{2+}$ mobilisation and CD28 costimulation in mature primary T cells. Eur. J. Immunol. 31:3567-3579. 